\title{
Sensitivity analysis for the study of influential parameters in tyre models
}

\section{Rimyalegdo KIEBRE}

University of Haute Alsace

12 rue des frères Lumière, 68093 Mulhouse cedex, France.

Phone: +33 (0)389336951Ｅ-mail: rimyalegdo.kiebre@uha.fr

\section{Floriane ANSTETT-COLLIN}

University of Haute Alsace

12 rue des frères Lumière, 68093 Mulhouse cedex, France.

E-mail: floriane.collin@uha.fr

\section{Michel BASSET}

University of Haute Alsace

12 rue des frères Lumière, 68093 Mulhouse cedex, France.

E-mail: michel.basset@uha.fr

\begin{abstract}
This paper studies two tyre models, the Fiala model and the Pacejka model. Both models are nonlinear and depend on parameters which must be identified from measurement data. A major problem is to efficiently prepare and plan the experiments. It is necessary to determine the parameters which have the greatest influence on the model output, and account for the output uncertainty which must be reduced. Therefore, the methodology presented here will help to carry out a variance-based global sensitivity analysis on both tyre models. This type of sensitivity analysis consists in studying and quantifying the contribution of each parameter to the total variance of the model output. Each parameter is classified into a hierarchy with respect to the importance of its influence. Fiala's model depends on three parameters and it is shown that only two have a significant influence on the model output. In Pacejka's model, which depends on seven parameters, the analysis has shown that only three parameters have a significant influence on the output. So, to improve the accuracy of these model responses, it is necessary to pay particular attention to the measurement data which help to better determine the values of their influential parameters.
\end{abstract}

Keywords: Sensitivity analysis; influential parameters; nonlinear model; tyre models; model uncertainty.

Reference to this paper should be made as follows: KIEBRE, R., ANSTETT-COLLIN, F. and BASSET, M. (2010) 'Sensitivity analysis for the study of influential parameters in tyre models', Int. J. of Vehicle Systems Modelling and Testing, Vol. x, No. x, pp.xxx-xxx.

Copyright (c) 2009 Inderscience Enterprises Ltd. 
Biographical notes: Rimyalegdo KIEBRE received his M.Sc. degree in 2007 in Mechanical Engineering from Ecole Nationale Supérieure d'Ingénieurs Sud Alsace (ENSISA), Mulhouse, France. He is currently a Ph.D. student at University of Haute Alsace (UHA) and affiliated with the Modélisation Intelligence Processus Systèmes (MIPS) laboratory, Mulhouse, France. His Ph.D. programme includes modelling of aircraft tyre/road interface characteristics and analysis of aircraft landing gear dynamics.

Floriane Anstett-Collin received her Ph.D. degree in automatic control theory from Nancy University, France, in 2006. She is actually assistant professor at the University of Haute Alsace and affiliated with the Modélisation Intelligence Processus Systèmes Laboratory, Mulhouse, France. Her research interests are modelling, identification and sensitivity analysis of complex systems. The main application is in the automotive and aeronautical domains.

Michel Basset received his Ph.D. degree in automatic control, from University of Haute Alsace, Mulhouse, France, in 1991. He is currently professor at University of Haute Alsace (UHA) and affiliated with the Modélisation Intelligence Processus Systèmes (MIPS) Laboratory. His research activities include measurement, modelling and control of continuous complex dynamic systems with applications in the automotive domain. $\mathrm{He}$ is also an active member of the IFAC Automotive Control Technical Committee.

\section{Introduction}

In the automotive and aeronautical fields, modelling the tyre/road interface is fundamental. Indeed, the tyre model is one of the major elements to integrate into a ground vehicle or aircraft model, as the tyres are the only contact points with the road. In general, the tyre models available in the literature, for example [Pacejka (2006); Rajamani (2005); Gipseret et al. (1997); Blundell et al. (1994)], are nonlinear and complex. They depend on a certain number of parameters obtained from experimental data. These measurement data are often few or/and uncomplete, especially in the aircraft domain, and involve high costs. A number of parameters are estimated with more or less precision, which often leads to unacceptable uncertainty on the model output. Among all the parameters, however, some only have a small or insignificant influence on the model response and therefore, do not need to be determined precisely. On the other hand, some parameters are determinant for the model response and thus influence its uncertainty significantly. These parameters may require additional measurement data in order to be estimated with relatively high accuracy. In order to prepare and plan the experiments, it is necessary to distinguish the parameters with an insignificant influence on the response uncertainty, so as to set them at their nominal value in their interval of variation, thanks to the sensitivity analysis. Different approaches have been proposed in the literature, for sensitivity analyses 
of nonlinear models [Cukier et al. (1997); Sobol (1993); Saltelli et al. (1999, 2000); Frey et al. (2002); Saltelli (2002); Saltelli et al. (2004); Jacques et al. (2006)]. There are qualitative [Saltelli et al. (2000)] and quantitative approaches [Saltelli et al. (1999)]. The qualitative approaches help to classify the parameters into a hierarchy with respect to the importance of their influence on the model response. The quantitative approaches are constructive in the sense that they not only allow the classification of the parameters into a hierarchy but also provide the means for the quantification of each parameter influence, contrary to the qualitative approaches. The present study focuses exclusively on the quantitative approaches. There are two types of quantitative approaches: local and global. Local approaches help to determine the impact of a small parameter variation around a nominal value. This impact is determined by calculating the partial derivative of the output function vs the corresponding parameter at the nominal value [Turyani et al. (2000)]. The global approaches also allow the determination of the same impact but by varying the parameter in its entire range of variation. These methods are based on the analysis of the output variance [Sobol (1993); Saltelli et al. (1999, 2004)]. They consist in calculating the contribution of the individual parameter to the total variance of the model output. Each parameter has two types of contribution to the total output variance. On the one hand, there is the main contribution of the parameter, which corresponds to the contribution of the parameter alone. On the other hand, there is the collective contribution of the parameter, which represents the influence of its interactions with the other parameters. The total contribution of the parameter is the sum of its main and collective contributions.

The aim of this paper is first to provide a methodology for sensitivity analysis, then to carry out a variance-based global sensitivity analysis on two tyre models, the Fiala model [Blundell et al. (1994))] and the Pacejka model, which is a basic function of the Magic Formula [Pacejka (2006)]. The study focuses on these two models as they are part of a project at the MIPS (Modélisation Intelligence Processus Systèmes) laboratory. In addition, Pacejka's model is widely used in the automotive and aeronautical fields. Both models depend on parameters (such as the friction coefficient, cornering stiffness, ...) which must be identified from measurement data. The parameters responsible for the observed uncertainty are then determined.

This study is organized as follows. Section 2 presents the main objectives of the sensitivity analysis and the proposed approach, followed by the definition of the sensitivity indices and a method for their estimation. The tyre models are studied in section 3 and a variance-based sensitivity analysis is carried out. For each model, the influence of the parameters is quantified and the most influential parameters are highlighted.

\section{Sensitivity analysis}

The main objectives of a sensitivity analysis can be summarized as follows.

- To attest the reliability of or the confidence in the model prediction. For example, if the sensitivity analysis shows a high influence of a parameter 
which in reality is known to have little (or no) influence, then the model is not reliable and should be modified.

- To determine the parameters which have the greatest influence on the total output variance. These parameters are responsible for the uncertainty of the output. So, the quality of the output can be increased by reducing the error in the most influential parameters.

- To identify the parameters which have little or no influence on the total variance of the model output. These parameters can be set at their nominal values, which will reduce the number of parameters (and also reduce the model complexity) with no significant effect on its accuracy.

- To determine which parameters interact with the others. In fact, a parameter on its own may not be influential, while its interactions with the others have a greater importance in the model response.

Consider a mathematical model in its general form:

$$
M: y=f\left(x_{1}, \ldots, x_{n}\right)
$$

where $y \in \mathbb{R}$ represents the model output and $x_{i} \in \mathbb{R}, i=1, \ldots, n$ the $\mathrm{n}$ unknown parameters, which are considered as random and independent variables. The function $f$ is nonlinear with respect to $x_{i}$. Performing a sensitivity analysis on model $M$ allows the identification of the parameters $x_{i}$ which have the greatest influence on its response $y$. The approach describing the steps necessary to carry out a sensitivity analysis is presented, below.

\subsection{Description of the sensitivity analysis approach}

Performing a sensitivity analysis requires the following steps.

a) Definition of the model

This first step consists in clearly determining the output $y$ of the model $M$ as a function of the predefined parameters $x_{i}$.

b) Assignment of the variation limits and probability density function

Each parameter $x_{i}$ is considered as a random variable. It is then necessary to determine the variation limits of each parameter $x_{i}$ and to estimate the most probable value of the parameter within these limits in order to choose the most appropriate probability density function. The variation limits as well as the probability density function may depend on various issues such as physical, technical and economical possibilities or/and limitations. The probability density functions most often considered are normal, lognormal and uniform distributions. 
c) Generation of input vectors

This step consists in choosing an appropriate sampling method for generating the possible values for each parameter $x_{i}$ within its limits of variation and according to its distribution function during simulation. Among the sampling procedures, one can distinguish random sampling, quasi-random sampling and Latin hypercube sampling [Helton et al. (2002, 2005, 2006)]. As mentioned in [Helton et al. (2006)], Latin hypercube sampling may be an appropriate method for computationally demanding models. When it is not the case, random sampling may be considered.

d) Determination of the output distribution

For each sample value of the parameters $x_{i}$, the output $y$ is calculated. It is then possible to have an overview on the model uncertainty by plotting (for example) the histogram of frequencies as well as the polygon of cumulative frequencies for $y$.

e) Calculation of the sensitivity indices

The final step consists in evaluating quantitatively the influence of the parameters $x_{i}$ on the output $y$, by calculating the sensitivity indices.

The next subsection presents the definition of the sensitivity indices based on the analysis of the output variance.

\subsection{Sensitivity indices}

Consider the model $M$ described by equation (1). In order to appreciate the contribution of the parameter $x_{i}$ to the total variance of the output $y$, it is necessary to calculate the conditional variance of $y$ while setting $x_{i}=x_{i}^{*}$, denoted $V\left(y \mid x_{i}\right)$. Since the true value $x_{i}^{*}$ of $x_{i}$ is not known, the expectation of $V\left(y \mid x_{i}\right)$ for all possible values in the interval of variation of $x_{i}$ is considered instead. This expectation is denoted $E\left(V\left(y \mid x_{i}\right)\right)$. The greater the contribution of parameter $x_{i}$ to the total variance of $y$ is, the lower quantity $E\left(V\left(y \mid x_{i}\right)\right)$ will be. The total variance of $y$, denoted $V(y)$, can be expressed as a function of the expectation $E\left(V\left(y \mid x_{i}\right)\right)$. $V(y)$ then becomes:

$$
V(y)=V\left(E\left(y \mid x_{i}\right)\right)+E\left(V\left(y \mid x_{i}\right)\right)
$$

where the term $V\left(E\left(y \mid x_{i}\right)\right)$ is the variance of the conditional expectation of $y$ when $x_{i}$ is set ( $x_{i}$ supposed known). This term is also an indicator of the influence of $x_{i}$ on the total variance of $y$. Indeed, the more parameter $x_{i}$ contributes to the total variance of $y$, the greater quantity $V\left(E\left(y \mid x_{i}\right)\right)$ will be. In order to use a normalized indicator, the sensitivity index of parameter $x_{i}$, denoted $S_{i}$ [Archer et al. (1997); Saltelli et al. (2008)], is defined as follows:

$$
S_{i}=\frac{V\left(E\left(y \mid x_{i}\right)\right)}{V(y)}
$$

The value of the sensitivity index $S_{i}$ lies between 0 and 1 . The closer to 1 its value is, the more parameter $x_{i}$ contributes to the total variance of $y$. 
The sum of the sensitivity indices $S_{i}, i=1, \ldots, n$, associated to each parameter $x_{i}$ verifies the following relation:

$$
\sum_{i=1}^{n} S_{i} \leq 1
$$

Remark 2.1: If the model $M$ is additive, it can be re-expressed as follows:

$$
y=a_{0}+\sum_{i=1}^{n} f_{i}\left(x_{i}\right)
$$

where $a_{0}$ is a constant, the functions $f_{i}, i=1, \ldots, n$, are possibly nonlinear with respect to $x_{i}$. In the case of an additive model, the following expression holds:

$$
\sum_{i=1}^{n} S_{i}=1
$$

If model $M$ is nonlinear and non additive, the interactions between the different parameters will also influence the total variance of $y$. In this case, the sensitivity index $S_{i}$ is no longer the most appropriate indicator and the total sensitivity index, denoted $S_{T i}$ [Archer et al. (1997); Saltelli et al. (2008)], is preferred. The total sensitivity index is defined as:

$$
S_{T i}=1-\frac{V\left(E\left(y \mid x_{\sim i}\right)\right)}{V(y)}
$$

where the term $V\left(E\left(y \mid x_{\sim i}\right)\right)$ represents the variance of the conditional expectation of $y$ when all parameters are supposed known except parameter $x_{i}$. The total sensitivity index $S_{T i}$ includes the contribution due to parameter $x_{i}$ alone, which also corresponds to index $S_{i}$, and the contribution due to the interactions of $x_{i}$ with the other parameters. In [Sobol (1993)], it is demonstrated that the total variance of $y$ can be decomposed as:

$$
V(y)=\sum_{i=1}^{n} V_{i}+\sum_{i=1}^{n} \sum_{j=i+1}^{n} V_{i j}+\ldots+V_{12 \ldots n}
$$

where :

$$
\begin{aligned}
& V_{i}=V\left(E\left(y \mid x_{i}\right)\right) \\
& V_{i j}=V\left(E\left(y \mid x_{i}, x_{j}\right)-V_{i}-V_{j}\right. \\
& \vdots
\end{aligned}
$$

If equation (8) is divided by $V(y)$, the following relation is obtained:

$$
1=\sum_{i=1}^{n} S_{i}+\sum_{i=1}^{n} \sum_{j=i+1}^{n} S_{i j}+\ldots+S_{12 \ldots n}
$$


where $S_{i}$ represents the sensitivity index of parameter $x_{i}$ (contribution of $x_{i}$ alone to the total variance $V(y)$ ) and is often called first order sensitivity index. The terms $S_{i j}, \ldots, S_{12 \ldots n}$ are given by:

$$
\begin{aligned}
& S_{i j}=\frac{V_{i j}}{V(y)} \\
& \vdots \\
& S_{12 \ldots n}=\frac{V_{12 \ldots n}}{V(y)}
\end{aligned}
$$

The term $S_{i j}$ represents the contribution due to the interaction of parameter $x_{i}$ with parameter $x_{j}$ and is called second order sensitivity index. In the same manner, the term $S_{12 \ldots n}$ represents the contribution due to the interaction of parameter $x_{i}$ with the remaining parameters and is called $n$ order sensitivity index. Thus, the total sensitivity index $S_{T i}$ of parameter $x_{i}$ is expressed as:

$$
S_{T i}=S_{i}+\sum_{\substack{j=1 \\ j \neq i}}^{n} S_{i j}+\sum_{\substack{j=1 \\ j \neq i}}^{n} \sum_{\substack{k=j+1 \\ k \neq i \\ j \neq i}}^{n} S_{i j k}+\ldots+S_{i j k \ldots n}
$$

The sensitivity indices presented previously are often calculated analytically when the function $f$ of model $M$ is known and relatively simple. However, some models may be complex with a high number of parameters so that analytical calculations of the sensitivity indices become time consuming or even sometimes impossible. It is therefore necessary to estimate them. The following subsection presents a method of estimating the sensitivity indices, often referred to as the Monte Carlo method.

\subsection{Estimation of the sensitivity indices}

Several methods of estimating the sensitivity indices are available in the literature [Cukier et al. (1997); Sobol (1993); Schwieger (2004); Jacques et al. (2006); Mara et al. (2008)]. The approach used in this study is based on the Monte Carlo method [Sobol (1993)]. Consider a random sample of size $N$ for each parameter of model $M$ and suppose that $x_{i}^{k}$ denotes the $k$ th value of parameter $x_{i}$ in its random sample. The estimated value of the expectation of $y$, denoted $\hat{E}(y)$, is given by:

$$
\hat{E}(y)=\frac{1}{N} \sum_{k=1}^{N} f\left(x_{1}^{k}, \ldots, x_{n}^{k}\right)
$$

In the same manner, the estimated value of the variance of $y$, denoted $\hat{V}(y)$, is the following:

$$
\hat{V}(y)=\frac{1}{N} \sum_{k=1}^{N} f^{2}\left(x_{1}^{k}, \ldots, x_{n}^{k}\right)-(\hat{E}(y))^{2}
$$


In [Sobol (1993)], a technique for the estimation of the conditional variance of $y$ with respect to $x_{i}$ is proposed. It is based on the estimation of the expectation of $y$ and requires two samples of the same size $N$ for each parameter $x_{i}$. Suppose that $x_{i}^{k 1}$ denotes the $k$ th value of the parameter $x_{i}$ from sample 1 and $x_{i}^{k 2}$, the $k$ th value of parameter $x_{i}$ from sample 2 . The estimated value of the conditional variance of $y$ with respect to $x_{i}$, denoted $\hat{V}_{i}$, is the following [Sobol (1993)]:

$$
\hat{V}_{i}=\hat{U}_{i}-(\hat{E}(y))^{2}
$$

where $\hat{U}_{i}$ is given by the expression below:

$$
\begin{array}{r}
\hat{U}_{i}=\frac{1}{N} \sum_{k=1}^{N} f\left(x_{1}^{k 1}, \ldots, x_{i-1}^{k 1}, x_{i}^{k 1}, x_{i+1}^{k 1}, \ldots, x_{n}^{k 1}\right) \\
\quad \times f\left(x_{1}^{k 2}, \ldots, x_{i-1}^{k 2}, x_{i}^{k 1}, x_{i+1}^{k 2}, \ldots, x_{n}^{k 2}\right)
\end{array}
$$

The estimated value of the first order sensitivity index, denoted $\hat{S}_{i}$, is then obtained:

$$
\hat{S}_{i}=\frac{\hat{V}_{i}}{\hat{V}(y)}
$$

Moreover, the estimated value of the second order sensitivity index, $\hat{S}_{i j}$, is:

$$
\hat{S}_{i j}=\frac{\hat{V}_{i j}}{\hat{V}(y)}
$$

where $\hat{V}_{i j}$ is given by:

$$
\hat{V}_{i j}=\hat{U}_{i j}-(\hat{E}(y))^{2}-\hat{V}_{i}-\hat{V}_{j}
$$

The term $\hat{U}_{i j}$ is the estimated value of the conditional expectation of $y$ and is obtained by varying all its parameters except $x_{i}$ and $x_{j}$ :

$$
\begin{aligned}
& \hat{U}_{i j}=\frac{1}{N} \sum_{k=1}^{N} \ldots \\
& \ldots f\left(x_{1}^{k 1}, \ldots, x_{i-1}^{k 1}, x_{i}^{k 1}, x_{i+1}^{k 1}, \ldots, x_{j-1}^{k 1}, x_{j}^{k 1}, x_{j+1}^{k 1}, \ldots, x_{n}^{k 1}\right) \\
& \times f\left(x_{1}^{k 2}, \ldots, x_{i-1}^{k 2}, x_{i}^{k 1}, x_{i+1}^{k 2}, \ldots, x_{j-1}^{k 2}, x_{j}^{k 1}, x_{j+1}^{k 2}, \ldots, x_{n}^{k 2}\right)
\end{aligned}
$$

The same method is used to estimate the sensitivity indices of a higher order. Finally, the total sensitivity index of $x_{i}$ is expressed as:

$$
\hat{S}_{T i}=1-\frac{\hat{V}_{\sim i}}{\hat{V}(y)}
$$


where $\hat{V}_{\sim i}$ is the estimated conditional variance of $y$ with respect to all parameters except $x_{i}$. It also means that $\hat{V}_{\sim i}$ is estimated by varying only $x_{i}$ while keeping all the other parameters constant. $\hat{V}_{\sim i}$ is given by:

$$
\begin{aligned}
& \hat{V}_{\sim i}=\hat{U}_{\sim i}-(\hat{E}(y))^{2} \\
& \hat{U}_{\sim i}=\frac{1}{N} \sum_{k=1}^{N} f\left(x_{1}^{k 1}, \ldots, x_{i-1}^{k 1}, x_{i}^{k 1}, x_{i+1}^{k 1}, \ldots, x_{n}^{k 1}\right) \\
& \quad \times f\left(x_{1}^{k 1}, \ldots, x_{i-1}^{k 1}, x_{i}^{k 2}, x_{i+1}^{k 1}, \ldots, x_{n}^{k 1}\right)
\end{aligned}
$$

In the next section, these sensitivity indices are determined for two tyre models in order to determine their most influential parameters.

\section{Application of the sensitivity analysis to tyre models}

When a vehicle equipped with tyres is manoeuvred on the ground, the tyres are submitted to a number of forces. For example, a longitudinal force is developed when driving or braking torque is applied to the wheel. A lateral force appears when the wheel is at an angle or when it is steered to turn at a corner. The two models considered in this study take account of the longitudinal force (driving or braking force) and the lateral force, but the study will exclusively focus on each model expression for the lateral force developed in the case of a cornering manoeuvre in steady-state condition. The measurement data used here are of the aircraft domain. Besides, the parameters of each model considered are assumed independent.

\subsection{Fiala's model}

During cornering with no driving/braking torque or turn slip (the tyre is also said to be in pure cornering condition), the steady-state lateral force $F_{y}$ is expressed as:

$$
F_{y}=-\mu\left|F_{z}\right|\left(1-\left(1-\frac{C_{\alpha}|\tan \alpha|}{3 \mu\left|F_{z}\right|}\right)^{3}\right) \operatorname{sgn}(\alpha)
$$

where $C_{\alpha}$ is the cornering stiffness, $F_{z}$ the vertical load $\left(F_{z}>0\right)$ and $\mu$ the lateral friction coefficient. Quantity $\alpha$ is the angle between the wheel plane and the wheel direction of motion. It is also called side slip angle. The term $\operatorname{sgn}(\alpha)$ represents the sign of the side slip angle $\alpha$. A sensitivity analysis is performed on the Fiala model. The approach presented in section 2.1 is applied to complete this study.

a) Definition of the model

The lateral force $F_{y}$ represents the model output. Within the framework of the MIPS project, the vertical load $F_{z}$ is measured and thus considered as known. It will be fixed at $F_{z}=90 \mathrm{kN}$, the nominal value used in the present project. Parameters $C_{\alpha}, \mu$ and $\alpha$ are unknown and their values are 
determined from measurement data. Therefore, they are the parameters whose influence should be studied.

b) Assignment of the variation limits and probability density function In pure cornering condition, the variation limits of $C_{\alpha}$ are estimated using a database, which considers an interpolation function similar to that presented in [Pacejka (2006)] and which gives $C_{\alpha}$ as a function of the vertical load $F_{z}$ and the tyre lateral stiffness. The variation interval of $C_{\alpha}$ is estimated to be $[179.02 \mathrm{kN} / \mathrm{rad} ; 1165 \mathrm{kN} / \mathrm{rad}]$. The cornering stiffness $C_{\alpha}$ also represents the derivative of the lateral force $F_{y}$ vs the side slip angle $\alpha$ at $\alpha=0$ :

$$
C_{\alpha}=\left.\frac{\partial F_{y}}{\partial \alpha}\right|_{\alpha=0}
$$

The value of $C_{\alpha}$ strongly depends on the tyre lateral stiffness. In fact, it may be considered that the stiffer the tyre carcass is, the greater $C_{\alpha}$ will be independently of the friction state in the tyre/road interface.

Then, the maximum value $F_{y \max }$ which the lateral force can reach, depends on the tyre/road contact state and is most often assumed to be proportional to the maximum value of the friction coefficient, $F_{y \max }=\mu_{\max } F_{z}$. The value of $\mu_{\max }$ corresponds to the upper limit of the friction coefficient interval. In the present case, it is supposed that $\mu_{\max }=1$, which is simply based on the Coulomb's friction law and means that the maximum value which the lateral force can reach is $90 \mathrm{kN} . \mu$ is assumed to vary in the interval $[0.4 ; 1]$. However, for some specific tyres, $\mu_{\max }$ can be greater than 1 and as a result, the importance of $\mu$ in the output would be significantly different, [Kiebre et al. (2010)].

Finally, parameter $\alpha$ is supposed to vary in the interval $\left[0 ; 20^{\circ}\right]([0 ; 0.35 \mathrm{rad}])$. It can be mentioned that, for most civil aircraft tyres, the maximum lateral force is commonly observed for side slip angle beyond $14^{\circ}$ (dry road condition) and may reach up to $20^{\circ}$ with respect to the road and vertical load conditions.

It is worth noting that the limits of variation determined for each parameter also take account of a database for aircraft tyres. Moreover, parameters $C_{\alpha}$, $\mu$ and $\alpha$ are assumed independent. They are deemed to follow a uniform distribution law in their corresponding interval.

Remark 3.1: Simulations with a normal distribution law for each parameter within its range of variation, not presented here, were also performed. The simulation results show the same trend in the distribution of the output and the sensitivity indices are not significantly different from those obtained under assumption of a uniform distribution law. It seems that the variation limits of the parameter are more influential than the distribution itself.

c) Generation of the parameters

The model presented in equation (23) is not computationally demanding 
and so, random sampling has been adopted (function rand from Matlab software). A sample size of $N=100000$ is considered for each parameter.

Remark 3.2: $\quad$ Tests with smaller sample sizes $(N=10000, N=20000$ and $N=50000$ ), not presented in this document, have also been considered. It must be noted that the results do not vary significantly. However, further investigations could help to determine the sample size(s) which lead(s) to a better compromise between precision and computation time.

d) Determination of the output distribution

The output $F_{y}$ is generated according to equation (23) and using the previous samples for $\mu, C_{\alpha}$ and $\alpha$. Figure 1(a) presents the histogram of frequencies for the lateral force $F_{y}$ with respect to its classes. Figure $1(\mathrm{~b})$ presents the polygon of cumulative frequencies for $F_{y}$, which has been normalized.

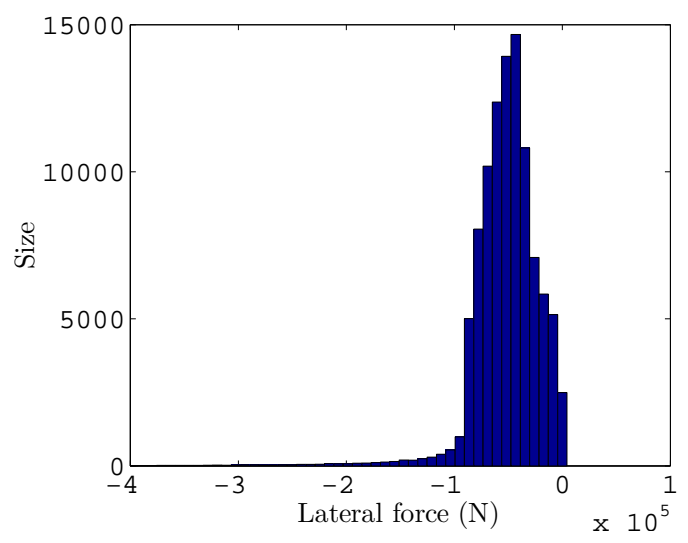

(a)

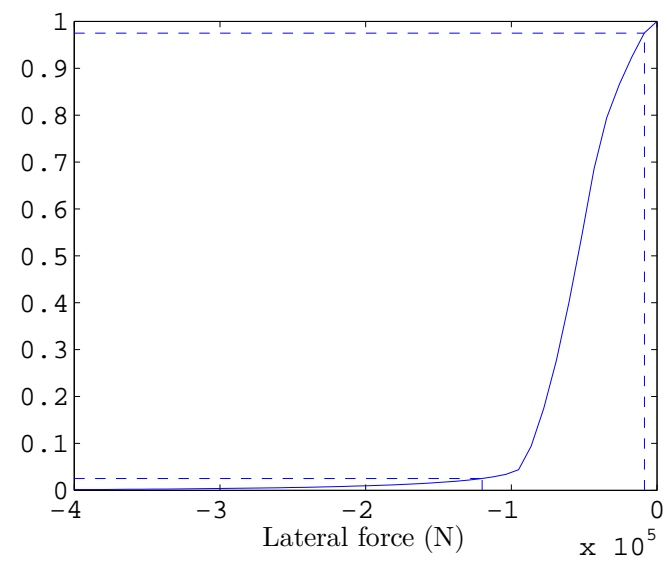

(b)

Figure 1 Fiala's model: (a) Histogram of frequencies - (b) Polygon of cumulative frequencies

The mean value of $F_{y}$ is $-52.85 \mathrm{kN}$ and its $95 \%$ confidence interval is [$120.95 \mathrm{kN} ;-8.64 \mathrm{kN}$ ], revealing an uncertainty of $112.3 \mathrm{kN}$ which represents $212 \%$ of the mean value of $F_{y}$. The objective is to estimate the contribution of $\alpha, \mu$ and $C_{\alpha}$ to this uncertainty.

e) Calculation of the sensitivity indices

Consider the lateral force $F_{y}$ given by equation (23). To estimate the sensitivity indices, two samples of the same size $N=100000$ are assumed for each parameter. Equations (13) and (14) are used to estimate the mean value of model $F_{y}, \hat{E}\left(F_{y}\right)=-52.85 \mathrm{kN}$, and its variance, $\hat{V}\left(F_{y}\right)=1.40 \times 10^{8}$, respectively. Then, the first order sensitivity indices, $S_{\mu}, S_{C_{\alpha}}$ and $S_{\alpha}$, are estimated using equation (17). 
The different indices are plotted in figure 2 and their corresponding values are given in table 1 .

\begin{tabular}{|c|c|}
\hline First order indices & Total indices \\
\hline$S_{\alpha}=0.348$ & $S_{T \alpha}=0.758$ \\
\hline$S_{C_{\alpha}}=0.1665$ & $S_{T C_{\alpha}}=0.5574$ \\
\hline$S_{\mu}=0.0166$ & $S_{T \mu}=0.3417$ \\
\hline
\end{tabular}

Table 1 Sensitivity indices for Fiala's model

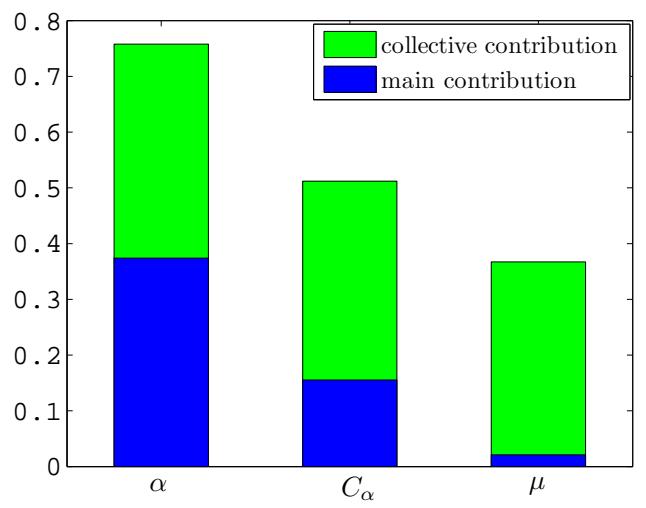

Figure 2 Sensitivity indices for Fiala's model

The first order index of the parameter $\alpha$ is the most important $\left(S_{\alpha}=0.348\right)$. So, the slip angle is the most influential parameter on the lateral force for the Fiala model. Parameter $C_{\alpha}$ follows $\left(S_{C_{\alpha}}=0.1665\right)$. Finally, the sensitivity index of $\mu$ is low $\left(S_{\mu}=0.0166\right)$. As a result, it can be underlined that when considering the influence of each parameter alone, only the side slip angle $\alpha$ and the cornering stiffness $C_{\alpha}$ have significant influence on the Fiala model response.

Moreover, the values of the total indices are higher than the ones of the first order indices. This is due to important interactions between all parameters which also influence the lateral force. Indeed, the sum of the first order indices is about $0.53\left(S_{\mu}+S_{C_{\alpha}}+S_{\alpha}=0.5311\right)$, which means that the contribution due to the interactions of parameters is as important as the individual contribution of the parameters.

In fact, the parameter $\mu$ determines the maximum value which the lateral 
force $F_{y}$ can reach. However, this maximum value of $F_{y}$ is also determined by a given value of the side slip angle $\alpha$. This explains the influence of the interaction between parameters $\mu$ and $\alpha$. On the other hand, parameter $C_{\alpha}$ determines the lateral deflection of the tyre. The influence of the parameter $C_{\alpha}$ on its own and of its interaction with the other parameters is not negligible as the lateral force is related to this tyre lateral deflection. In a word, for the Fiala model, the improved accuracy in the slip angle $\alpha$, in the cornering stiffness $C_{\alpha}$ and in the friction coefficient $\mu$ is necessary to reduce the uncertainty in the lateral force.

The next subsection presents the second model studied in this paper, the Pacejka model.

\subsection{Pacejka's model}

In pure cornering condition, an interpolation function, called Magic Formula, is proposed for the lateral force as presented in equation below:

$$
\begin{aligned}
F_{y}= & \mu F_{z} \sin \left[C \operatorname { a r c t a n } \left(\frac{K}{\mu F_{z} C}\left(\alpha+S_{h}\right)-E\left(\frac{K}{\mu F_{z} C}(\alpha\right.\right.\right. \\
& \left.\left.\left.\left.+S_{h}\right)-\arctan \left(\frac{K}{\mu F_{z} C}\left(\alpha+S_{h}\right)\right)\right)\right)\right]+S_{V}
\end{aligned}
$$

where $C, E, S_{h}$ and $S_{v}$ are empirical parameters. A sensitivity analysis of the Pacejka model is carried out using the proposed approach.

a) Definition of the model

The lateral force $F_{y}$ presented in equation (25) is the model output. For the same reason as in the Fiala model, $F_{z}$ is considered known and fixed at $90 \mathrm{kN}$. Parameters $\mu, \alpha, C, C_{\alpha}, E, S_{h}$ and $S_{v}$ are unknown and are under study.

b) Assignment of the variation limits and probability density function

Based on the tyre database, the variation limits of $C, E, S_{h}$ and $S_{v}$ are estimated by the following intervals: $[1 ; 2],[-3 ; 0.5],[-0.0037 \mathrm{rad} ; 0.0037 \mathrm{rad}]$ and $[-3322 \mathrm{~N} ; 3322 \mathrm{~N}]$ respectively. Similarly to the case of the Fiala model, the variation limits of parameters $\alpha, \mu$ and $C_{\alpha}$ are $\left[0 ; 20^{\circ}\right],[0.4 ; 1]$ and $[179.02 \mathrm{kN} / \mathrm{rad} ; 1165 \mathrm{kN} / \mathrm{rad}]$, respectively. A uniform distribution law is assumed for parameters $\mu, C, C_{\alpha}, E, S_{h}$ and $S_{v}$ in their corresponding range of variation. As in the Fiala model, a normal distribution law is also assumed for each parameter and the simulation results do not show a significant difference from those obtained under the assumption of a uniform distribution law.

c) Generation of input vectors

Pacejka's model as presented here is not computationally demanding. So, random sampling has been adopted (function rand from Matlab software). A 
sample size of $N=100000$ is considered for each parameter. As previously, tests with smaller sample sizes $(N=10000, N=20000$ and $N=50000)$, not presented in this document, have also been used and similar results obtained.

d) Determination of the output distribution

Output $F_{y}$ is generated according to equation (25) using the samples for the parameters. Figure 3(a) presents the histogram of frequencies for the lateral force $F_{y}$, with respect to its classes. Figure $3(\mathrm{~b})$ presents the polygon of cumulative frequencies for $F_{y}$ which has been normalized.

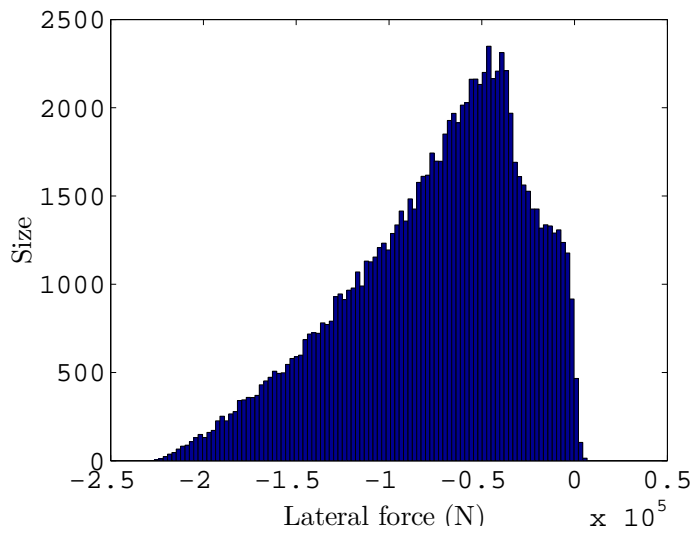

(a)

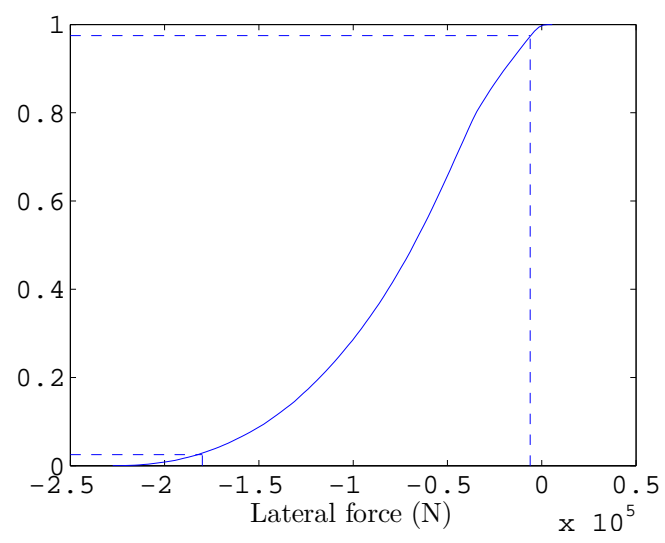

(b)

Figure 3 Pacejka's model: (a) Histogram of frequencies - (b) Polygon of cumulative frequencies

The mean value of $F_{y}$ is $-75.07 \mathrm{kN}$ and its $95 \%$ confidence interval is [$180 \mathrm{kN} ;-6.14 \mathrm{kN}$ ], indicating an uncertainty of $173.85 \mathrm{kN}$ equivalent to $231 \%$ of the mean value of $F_{y}$. The objective is to classify all the parameters according to their contribution to this uncertainty.

e) Calculation of the sensitivity indices

Assume the expression of the lateral force $F_{y}$ as described by equation (25). Two samples of the same size $N=100000$ are considered for each parameter. The estimated mean value of $F_{y}$ is $\hat{E}\left(F_{y}\right)=-75.07 \mathrm{kN}$ (equation (13)) and its estimated variance is $\hat{V}\left(F_{y}\right)=2.23 \times 10^{9}$ (equation (14)). Equation (17) is used to estimate the first order indices $S_{\alpha}, S_{\mu}, S_{C_{\alpha}}, S_{C}, S_{E}, S_{S_{h}}$ and $S_{S_{v}}$. The total sensitivity indices $S_{T \alpha}, S_{T \mu}, S_{T C_{\alpha}}, S_{T C}, S_{T E}, S_{T S_{h}}$ and $S_{T S_{v}}$ are estimated using equation (21). The different indices are plotted in figure 4 and their corresponding values are given in table 2 . 


\begin{tabular}{|c|c|}
\hline First order indices & Total indices \\
\hline$S_{\alpha}=0.2033$ & $S_{T \alpha}=0.5867$ \\
\hline$S_{\mu}=0.1883$ & $S_{T \mu}=0.3661$ \\
\hline$S_{C_{\alpha}}=0.1609$ & $S_{T C_{\alpha}}=0.2657$ \\
\hline$S_{E}=\simeq 0$ & $S_{T E}=0.0072$ \\
\hline$S_{C} \simeq 0$ & $S_{T C}=0.0035$ \\
\hline$S_{S_{v}} \simeq 0$ & $S_{T S_{v}}=0.0017$ \\
\hline$S_{S_{h}} \simeq 0$ & $S_{T S_{h}} \simeq 0$ \\
\hline
\end{tabular}

Table 2 Sensitivity indices for Pacejka's model

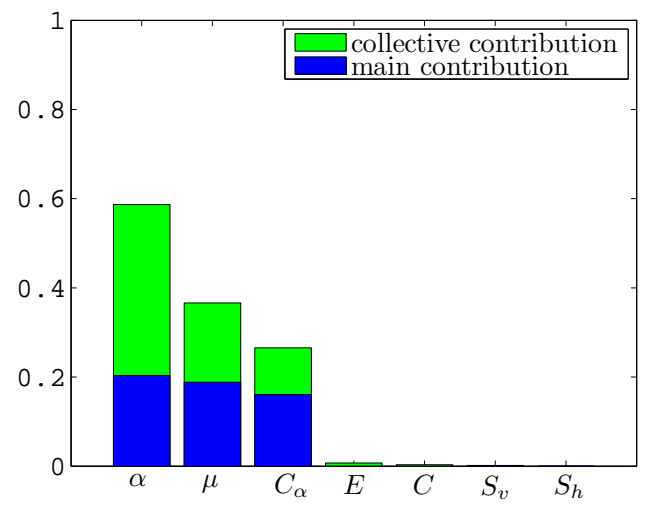

Figure 4 Sensitivity indices for Pacejka's model

Figure 4 allows the different parameters to be classified into a hierarchy according to their influence on the total variance of the lateral force. It is shown that parameter $\alpha$ has the highest sensitivity index $\left(S_{\alpha}=0.2033\right)$. Therefore, this parameter is the most influential. However, the index of $\mu$ has a value close to that of $\alpha\left(S_{\mu}=0.1883\right)$. Parameter $C_{\alpha}$ follows $\left(S_{C_{\alpha}}=\right.$ $0.1609)$. Finally, parameters $C, E, S_{v}$ and $S_{h}$ have their sensitivity indices close to 0 . Therefore, their influence on the total variance of the model response can be considered negligible. Moreover, the sum of the first order sensitivity indices of the parameters is 0.5525. As in the Fiala model, the interactions between all the parameters also influence the lateral force.

For the Pacejka model, parameters $\alpha, C_{\alpha}$ and $\mu$ have the same physical 
meaning for the tyre as mentioned previously for Fiala's model. However, in Pacejka's model, the sensitivity indices of $\alpha, C_{\alpha}$ and $\mu$ are quite close to each other, contrary to the Fiala model. Thus, the interpolation function used in the Pacejka model gives almost the same importance to these three parameters. $C, E, S_{v}$ and $S_{h}$ are empirical scaling parameters used for adjusting the curve shape and reducing the interpolation error. This might be the reason why their individual influence is negligible.

To conclude, in the Pacejka model, only three parameters, the slip angle $\alpha$, the friction coefficient $\mu$ and the cornering stiffness $C_{\alpha}$, significantly influence the lateral force. Consequently, improving their accuracy would help to considerably improve the uncertainty in the lateral force $F_{y}$. Concerning parameters $C, E, S_{v}$ and $S_{h}$, each one can be fixed at its nominal value in the corresponding interval of variation with no significant effect on the accuracy of the model response.

\section{Conclusion}

A methodology for sensitivity analysis has been presented. A variance-based global sensitivity analysis has been carried out on two models which describe the lateral force occurring at the tyre/road interface. This sensitivity analysis consists in quantifying the contribution of each parameter to the total variance of the model output. The data used are of the aircraft domain. In the Fiala model, the lateral force depends on three parameters, the side slip angle, the friction coefficient and the cornering stiffness. It has been shown that, when considering each parameter alone, only the side slip angle and the cornering stiffness influence the lateral force. However, the interaction between the three parameters have also an important influence on the model response. Therefore, improving the accuracy of these three parameters would help to reduce the uncertainty of the model response. In the case of the Pacejka model, the lateral force expression depends on seven parameters. However, only three of them, the side slip angle, the friction coefficient and the cornering stiffness, influence the lateral force significantly, in the same order. Improving the accuracy of these three parameters would help to considerably improve the uncertainty in the lateral force. The parameters of both models studied are assumed independent. However, tyre models often include correlated parameters. So, further studies on methods of sensitivity analysis for models with correlated parameters may be necessary. It would also be interesting to compare the results of this method (convergence of the sensitivity indices and the computation time) with those obtained with other methods of sensitivity analysis (McKay, FAST, ...).

\section{References}

Pacejka, H.B. (2006) Tyre and Vehicle Dynamics, Elsevier, ISBN 0750669187.

Blundell, M. and Harty, D. (2005) Multibody systems approach to vehicle dynamics, Elsevier.

Rajamani, R. (2005) Vehicle dynamics and control, Springer Verlag, ISBN 0387263969. 
Gipser, M. and Hofer, R. , and Lugner, P. (1997) Dynamical tyre forces response to road unevennesses, Vehicle System Dynamics Vol. 27, pp. 94-108.

Cukier, R.I., Levine, R.I. and Shuler, K.E. (1978) Nonlinear sensitivity analysis of multiparameter model systems, Journal Computational Physics, vol. 26, pp. 1-42.

Saltelli, A., Chan, K. and Scott, E.M. (2000) Sensitivity Analysis. John Wiley and Sons, Chichester.

Frey, H. C. and Patil, S. R. (2002) Identification and review of sensitivity analysis methods. Risk analysis, vol. 22, n3, pp. 553-578.

Saltelli, A. (2002) Sensitivity analysis for importance assessment. Risk Analysis, vol. 22, n3.

Saltelli, A., Tarantola, S., Campolongo, F. and Ratto, M. (2004) Sensitivity analysis in practice. John Wiley and Sons, Chichester, 2004.

Saltelli, A., Tarantola, S. and Chan, K. (1999) A quantitative model independent method for global sensitivity analysis of model output. Technometrics, vol. 41, pp. 39-56.

Saltelli, A., Ratto, M., Andres, T., Campolongo, F., Cariboni, J., Gatelli, D. Saisana, M. and Tarantola, S. (2008) Global sensitivity analysis, The Primer. John Wiley and Sons.

Turyani, T. and Rabitz, H. (2000) Local methods in Sensitivity analysis, eds : Saltelli A., Chan K., Scott E. M. John Wiley and Sons, Chichester.

Sobol, I. M. (1993) Sensitivity estimates for nonlinear mathematical models. Mathematical Modelling and Computational Experiments, vol. 1, pp. 407-414.

McKay, M. D., Morrison, J. D. and Upton, S. C. (1999) Evaluating prediction uncertainty in simulation models. Computer Physics Communications, vol. 117, n12, pp. 44-51.

Jacques, J., Lavergne, C. and Devictor, N. (2006) Sensitivity Analysis in presence of Model Uncertainty and Correlated Inputs. Reliability Engineering and System Safety, vol. 91, pp. 1126-1134.

Schwieger, V. (2004) Variance-based sensitivity analysis for model evaluation in engineering surveys. Proceedings of 3rd International Conference on Engineering Surveying, Bratislava, Slovaquie, 11-13 novembre 2004.

Mara, T.A and Tarantola, S. (2008) Application of global sensitivity analysis of model output to building thermal simulations. Journal of Building Simulation, vol. 1, pp. 290-302.

Archer, G. E. B., Saltelli, A. and Sobol, I. M. (1997) Sensitivity measures, Anovalike techniques and the use of bootstrap. Journal of Statistical Computation and Simulation, vol. 58, pp. 99-120.

Helton, J.C., Johnson, J.D., Sallaberry, C.J. and Storlied, C.B. (2006) Survey of sampling-based methods for uncertainty and sensitivity analysis. textitReliability Engineering and System Safety, Vol. 91, pp. 1175-1209.

Helton, J.C., Davis, F.J. and Johnson, J.D. (2005) A comparison of uncertainty and sensitivity analysis results obtained with random and Latin hypercube sampling. Reliability Engineering and System Safety, Vol. 89, pp. 305-330.

Helton, J.C., and Davis, F.J. (2002) Illustration of Sampling-Based methods for uncertainty and sensitivity analysis, Risk Analysis, Vol. 22, No. 3.

Kiébré, R., Anstett-Collin, F., Basset, M. (2010) Analyse de sensibilité pour l'étude des paramètres influents dans les modèles d'interface pneu/sol. Conférence Internationale Francophone d'Automatique (CIFA 2010), Nancy, France, 2-4 of June, 2010 . 